doi:

\title{
YU.V. KOVTUN
}

National Science Center "Kharkiv Institute of Physics and Technology",

Nat. Acad. of Sci. of Ukraine

(1, Akademichna Str., Kharkiv 61108,Ukraine; e-mail: Ykovtun@kipt.kharkov.ua)

\section{ENERGY EXPENDITURE FOR WATER MOLECULE IONIZATION BY ELECTRON IMPACT IN WEAKLY IONIZED PLASMA}

\begin{abstract}
The energy balance of the water molecule ionization by a monoenergetic electron beam with the energy of primary electrons in the interval of 15-1000 eV has been calculated. The dependences of the ionization cost on the water ionization degree within the interval from 0 to 0.1 are obtained. The ionization cost is shown to increase with the ionization degree. In particular, for a primary electron energy of $1000 \mathrm{eV}$, it increases from 25.26 to $52.45 \mathrm{eV}$ in the examined ionization degree interval.
\end{abstract}

Ke ywords: ionization cost, ionization degree, water molecule, electrons.

\section{Introduction}

Researches of the physical and chemical processes running in water in various aggregate states, when water molecules interact with various particles (electrons, ions, photons, and so on), are of interest for tackling a wide range of physical and application issues. For instance, these are astrophysics (the study of planets in the solar system and their satellites, interstellar medium, etc.) [1,2], radiation physics, chemistry, biology, medicine (the study of the influence of ionizing radiation on biological objects and the application of the data obtained to develop methods for the diagnostics and treatment of diseases) [36], plasma physics (electric discharges in water and above its surface, as well as in water vapor) [7-14], and other domains of science and engineering.

One of the corresponding directions, which invokes a considerable interest in low-temperature plasma physics and its application technologies, includes researches of electric discharges in the liquid and above its surface. Regardless of a long, for more than a

(c) YU.V. KOVTUN, 2016

12 century, history of researches of the electric discharge in liquids, the modern studies are characterized by a large variety of experimental devices and a wide range of initial experimental conditions [8]. A large body of experimental data concerning the electric breakdown in liquids, which was obtained till now, testifies that there are a number of various breakdown mechanisms. According to work [15], the following four mechanisms of discharge initiation can be distinguished.

- Gas dissolved in a liquid (in the form of gas bubbles) plays a crucial role in the discharge ignition. Its ionization leads to the formation of primary plasma channels. This mechanism is most possibly realized in non-degassed liquids.

- At high electric field strengths near the electrode, electrons are emitted into the liquid (discharge from the cathode) or molecules in the liquid become ionized (discharge from the anode). As a result, the liquid is heated up, and a shock wave is formed. Behind the shock wave front, the explosive formation of vapor and the formation of a plasma channel by ionizing gas-vapor bubbles take place.

- If the electric field strength is high $\left(\sim 10^{8} \mathrm{~V} / \mathrm{cm}\right)$ and the pulse is short $\left(\sim 10^{-8} \mathrm{~s}\right)$, a plasma channel can

ISSN 2071-0186. Ukr. J. Phys. 2016. Vol. 61, No. 1 
be formed as a result of the ionization of molecules in the liquid because of the autoionization (anode initiation) or impact ionization (cathode initiation). In this case, the field strength is strong enough for the molecules in the liquid to be ionized. However, there is no enough time for a phase transition of the first kind to occur.

- In liquids with a high specific conductance, an electric current gives rise to the heating of a liquid, its boiling, ionization of gas-vapor cavities, and the formation of plasma channels.

While developing any technology, in which electric discharges in a liquid and above its surface will be used, the determination of main channels of energy expenditure and, accordingly, the total amount of energy expenditure comprises an important task. This issue is rather difficult, because, in the general case, this parameter depends on specific technological conditions. However, one of the main channels of energy expenditure - namely, to create plasma - can be determined in principle.

A number of processes running in plasma - such as the ionization, excitation, recombination, diffusion, recharging, and others - are, in essence, major factors that affect the final energy expenditure for the creation of a plasma with required parameters. One of them, electron impact ionization, determines the energy expenditure, which is called the ionization cost. This process can proceed in two ways: either by a direct transition of a bound electron into the continuum or by cascade transitions of an electron into the continuum. In other words, the collision of the electron with a particle can result in the direct dissociative step-like ionization and the autoionization.

Electron impact ionization is not a unique process when a neutral particle transits into an ionized state. Ionization owing to collisions between photons and particles (photoionization) is also possible. Photons are emitted in discharges by excited particles. The photoionization cross-section is several orders of magnitude smaller than that of electron impact ionization. Collisions of heavy particles (atoms and molecules) with other neutral or charged heavy particles can also give rise to the formation of ions. For colliding particles with high energies $(\mathrm{keV}$, $\mathrm{MeV}$ ), those processes play an appreciable role in the ionization balance, which is taken into account, e.g., while studying a high-temperature plasma at the controlled thermonuclear fusion. However, there are almost no high-energy heavy particles in electric discharges. But collisions between atoms (molecules) and neutral particles can be rather an effective mechanism of ion formation, e.g., in the course of Penning or associative ionization.

It is worth noting that, at electric discharges, the thermal ionization is possible as well. In this case, the plasma is supposed to be in the thermodynamic equilibrium state, or deviations from this state are rather small (the model of local thermodynamic equilibrium). The concentrations of electrons, ions, and neutrals are related to the temperature by the Saha equation, irrespective of the mechanisms of charge creation and annihilation. Recent researches of electric discharges of the arc, spark, glow, and other types $[8,9]$ in a liquid and above its surface demonstrated that, in many cases, the created plasma is not thermal [8].

It is also worth noting that negative ions, which can appear in the plasma of electric discharges, also participate in the particle balance in a plasma. Unfortunately, there is no comprehensive model till now, which would involve a considerable number of processes running in electric discharges in a liquid and above its surface $[8,9,15]$. All the processes mentioned above can prevail at certain discharge stages or make a substantial contribution to the ionization processes on the whole. As was marked in work [15], the electron impact ionization is one of the processes dominating at the liquid breakdown and the plasma formation. The authors of works [11-13] supposed that the main contribution to the plasma formation is brought by a group of fast electrons. In other words, the electron impact ionization is an unavoidable process at the plasma formation in discharges.

The term "electron impact ionization cost" is usually understood as a certain average energy spent by an electron to form an ion-electron pair in the medium. Of course, the magnitude of ionization cost cannot be lower than the ionization potential, which amounts to $12.621 \mathrm{eV}$ for water in the gas phase [16]. In the liquid phase, the ionization energy threshold is governed by various mechanisms of ionization such as the direct vertical transition (the FranckCondon model), autoionization, and others - and equals $8.5-10 \mathrm{eV}$ or even more, whereas the photoionization can occur at a level of the excitation energy of about $6.5 \mathrm{eV}$ [17]. Since the plasma channels are 
mainly formed by ionizing the gas-vapor phase [15], the ionization cost will be considered for water in the gaseous state.

The ionization cost can be determined both experimentally and theoretically. One of the theoretical approaches consists in the consideration of elementary processes that take place, when a monoenergetic electron flux (beam) passes through the medium. In this case, the energy of a primary electron is spent in elastic and inelastic collisions with a molecule. In addition, some energy is absorbed by an electron knocked out from the molecule in the course of ionization (dissociative ionization). The energy of this electron falls within the limits from 0 to $E-E_{\mathrm{iz}}$, where $E$ is the energy of a primary electron, and $E_{\mathrm{iz}}$ the ionization energy $[18,19]$. Some of the knocked out (secondary) electrons, the energies of which exceed $E_{\mathrm{iz}}$, also participate in the ionization process and, in turn, form a new generation of secondary electrons. What is actually considered is the total energy lost by the monoenergetic electron flux (beam) in the medium as a result of the ionization cascades. The average energy spent to form an ion-electron pair, i.e. the ionization cost, is determined by the formula [3]

$W=\frac{E}{\left\langle N_{i}\right\rangle}$,

where $\left\langle N_{i}\right\rangle$ is the average number of ions generated at the collisions of electrons with a molecule. As is seen from this equation, the determination of the $W$ value is reduced to the finding of the average number of ions that are formed at the collisions of electrons with a molecule. For this purpose, one should know the threshold energies and the cross-sections of elementary processes, which in turn affect the ultimate value of ionization cost, as was emphasized earlier, e.g., in work [6]. Accordingly, the calculation of the ionization cost always gives an approximation to the real value.

The application of this approach is quite justified when the main contribution to the medium ionization is made by the flux (beam) of primary electrons. Therefore, in principle, the latter can be used to determine the ionization cost of water at the initial stage of ionization of the corresponding gas-vapor phase. This is the more so because, in this approach, the influence of the ionization degree $n_{e} / n_{M}$, where $n_{e}$ is the concentration of electrons in plasma and $n_{M}$ the concentration of neutrals, on the ionization cost magnitude can be taken into account. Since the cross-section of Coulomb collisions is large, their contribution to the total ionization cost should become considerable, as the ionization degree increases. The corresponding calculations, in which the ionization degree of plasma was made allowance for, were carried out in a number of works for atomic $\mathrm{H}$, He [20], $\mathrm{O}$ [21], and $\mathrm{Ar}$ [22] gases, as well as molecular $\mathrm{H}_{2}$ $[20,23], \mathrm{CO}_{2}$ [24], $\mathrm{N}_{2}$ [25], and CO [26] ones.

Hence, it is of interest to calculate the electron impact ionization cost of a water molecule in the weakly ionized plasma. Such calculations are absent in the literature. This work continues our researches carried out earlier [11-14].

\section{Elementary Processes at Electron Collisions with Water Molecules}

The energy expenditure of an electron at collisions ultimately determines the magnitude of ionization cost. Therefore, let us consider the processes of electron collision with a water molecule in more details. The case of electron collision with a molecule, unlike that with an atom, is characterized by a large number of probable processes giving rise to energy losses by the incident electron $[18,19]$.

1. Elastic collisions of electrons with the molecule: $(e+A B \rightarrow e+A B)$. This process has no energy threshold, in contrast to other processes, and results in the momentum transfer to the molecule. It also strongly affects the scattering of electron beams at their passage through the medium. In this case, the average energy fraction lost at a collision equals $\frac{2 m_{e} \sigma_{\mathrm{mt}}}{m_{M} \sigma_{\text {elas }}}$, where $m_{e}$ is the electron mass, $m_{M}$ the molecule mass, $\sigma_{\mathrm{mt}}$ the transport (diffusive) crosssection of elastic collision, and $\sigma_{\text {elas }}$ the cross-section of elastic collision $[18,19]$. The cross-sections of elastic collisions were experimentally measured in a number of works. They were generalized and presented in the form of recommended data in works $[16,27]$.

2. Excitation of rotational-vibrational levels of the molecule: $\left(e+A B \rightarrow e+A B^{r v}\right)$. At inelastic collisions of an electron with the molecule, the kinetic energy transforms into the internal one. The total energy of the molecule is equal to the sum of three components: $E_{\text {tot }}=E_{\mathrm{el}}+E_{\mathrm{v}}+E_{\mathrm{r}}$, where $E_{\mathrm{el}}, E_{\mathrm{v}}$, and $E_{\mathrm{r}}$ are the energies of electron, vibrational, and rotational states, respectively. Therefore, the excitation of both rotational and rotational-vibrational levels is possi- 
Table 1

\begin{tabular}{|c|c|c|c|}
\hline Molecule, molecular ion & Electron configuration & Electron state & Transition energy, eV \\
\hline $\mathrm{H}_{2} \mathrm{O}$ & $\left(1 a_{1}\right)^{2}\left(2 a_{1}\right)^{2}\left(1 b_{2}\right)^{2}\left(3 a_{1}\right)^{2}\left(1 b_{1}\right)^{2}$ & $\begin{array}{c}X^{1} A_{1} \\
a^{3} B_{1} \\
A^{1} B_{1} \\
{ }^{3} A_{2} \\
{ }^{3} A_{1}\end{array}$ & \begin{tabular}{l}
\multicolumn{1}{c}{-} \\
7.14 \\
7.49 \\
8.9 \\
9.2
\end{tabular} \\
\hline $\mathrm{H}_{2} \mathrm{O}^{+}$ & $\left(1 a_{1}\right)^{2}\left(2 a_{1}\right)^{2}\left(1 b_{2}\right)^{2}\left(3 a_{1}\right)^{2}\left(1 b_{1}\right)^{1}$ & $\begin{array}{l}X^{2} B_{1} \\
A^{2} A_{1} \\
B^{2} B_{2}\end{array}$ & $\begin{array}{l}12.621 \\
13.748 \\
17.29\end{array}$ \\
\hline $\mathrm{H}_{2} \mathrm{O}^{+2}$ & $\left(1 a_{1}\right)^{2}\left(2 a_{1}\right)^{2}\left(1 b_{2}\right)^{2}\left(3 a_{1}\right)^{1}\left(1 b_{1}\right)^{1}$ & $\begin{array}{c}X^{3} B_{1} \\
1^{1} A_{1} \\
b^{1} B_{1} \\
2^{1} A_{1}\end{array}$ & $\begin{array}{l}40.2 \\
41.1 \\
42 \\
45\end{array}$ \\
\hline
\end{tabular}

ble, as well as the excitation of electron levels simultaneously with rotational and vibrational ones. The excitation of vibrational levels plays a substantial role in discharges activated in the molecular gas environment. This is one of the main mechanisms of energy transfer from electrons to molecules. However, the results of calculations carried out in work [28] testifies that the energy expenditure of electrons on the excitation of rotational levels at low electron energies prevails over the expenditure owing to elastic collisions. Of course, now it is impossible to consider all transitions in the water molecule. According to work [29], the latter has 12248 vibrational-rotational and 107 vibrational levels. At the same time, the set of data for level excitation cross-sections is incomplete. Therefore, only the data available in the literature and recommended for the rotational levels (the data calculated on the basis of the $R$-matrix method) $[16,28]$ and vibrational ones (the experimental data) $[16,30]$ were selected for calculations.

3. Excitation of electron levels of the molecule: $\left(e+A B \rightarrow e+A B^{*}\right)$. Electron transitions in the molecule can be activated by means of both internal (spontaneous radiation) and external (electron impact, radiation absorption) perturbations. The distance between the nuclei in the molecule and the velocity of their relative motion slightly change at that (the Franck-Condon principle) [19]. As a result, there may exist several scenarios of the electron transition depending on the shapes of the potential energy curves in the initial and final states. Experimentally measured excitation energies of 25 electron levels and, accordingly, the excitation cross-sections of those levels were taken from works [31-33]. Because of a small number of experimental points, the excitation crosssections were approximated by an empirical formula proposed in work [37]. In Table 1, the data of works $[16,33,35,36]$ concerning the transition energies for some lowest electron states in a water molecule and its molecular ions are summarized.

4. Dissociation of the molecule: $(e+A B \rightarrow e+$ $+A+B)$. At transitions accompanying by dissociation, according to the Franck-Condon principle, the minimum of the energy required for the transition equals [18]

$E_{\min }=E_{A}+E_{B}+D_{A B}+W_{\min }$,

where $E_{A}$ and $E_{B}$ are the total excitation energies of atoms $\mathrm{A}$ and $\mathrm{B}$, respectively, $D_{A B}$ the dissociation energy, and $W_{\min }$ the minimum kinetic energy of dissociation products. In essence, the minimum energy required for the transition, $E_{\min }$, is the threshold energy $E_{\mathrm{th}}$, below which the transition is impossible. The $\mathrm{H}_{2} \mathrm{O}$ molecule has the ground electron state $X^{1} A_{1}$ (see Table 1 ), and its dissociation energy equals $D(\mathrm{H}-\mathrm{OH})=5.0992 \mathrm{eV}[16]$. Excitation of the electron levels $a^{3} B_{1}, A^{1} B_{1}, b^{3} A_{1}$, and $B^{1} A_{1}$ results in the dissociation of a water molecule by several channels [37] into $\mathrm{H}$ and $\mathrm{OH}\left(X^{2} \Pi_{i}\right)$ with excited rotationalvibrational levels [38].

5. Ionization and dissociative ionization of the molecule: $\left(e+A B \rightarrow e+A B^{+}, e+A B \rightarrow e+A^{+}+B\right.$, $\left.e+A B \rightarrow e+A^{+}+B^{+}\right)$. The energy of formation of a 
molecular ion $\mathrm{H}_{2} \mathrm{O}^{+}$is equal to the energy of transition into the ground electron state (see Table 1). The process of dissociative ionization of a water molecule proceeds via several channels [39] characterized by different threshold energies $E_{\mathrm{th}}$. The fragments of the dissociative ionization can also include, besides neutrals, a few singly ionized particles, as well as doubly charged ones. A process, when more than one singly ionized particle is formed in fragments of the dissociative ionization, can occur both through the autoionization [40,41] and the formation of a doubly ionized molecule $\mathrm{H}_{2} \mathrm{O}^{2+}$ (see Table 1) followed by its subsequent decay [42]. The energy of ternary ionization of an $\mathrm{H}_{2} \mathrm{O}$ molecule, according to the data of works $[43,44]$, is at a level of $75 \mathrm{eV}$. According to the results of work [44], for the electron energy within the interval of 30-200 eV, the contribution of the multiple ionization of an $\mathrm{H}_{2} \mathrm{O}$ molecule to the total number of generated ions amounts to $0-4.8 \%$ at the double ionization, and to $0-0.09 \%$ at the triple one. The experimental values of ionization and dissociative ionization cross-sections were taken from work [27].

6. Dissociative attachment and polar dissociation: $\left(e+A B \rightarrow e+A^{-}+B, e+A B \rightarrow e+A^{+}+B^{-}\right)$. Dissociative attachment occurs by means of the electron capture by the molecule and the subsequent dissociation of the latter into a negative ion and a neutral [45]. This process plays a corresponding role in the balance of particles at low electron energies. However, since it results in electron losses together with recombination and diffusion, this process will not be taken into consideration below, while calculating the ionization cost. It should also be noted that those processes play an appreciable role in the balance of plasma particles and affect the ultimate value of energy expenditure for the plasma creation. Polar dissociation [39] will also be neglected, because the cross-section of this process is small in comparison with the ionization (dissociative ionization) cross-section, e.g., for oxygen and carbon oxide [18]. Moreover, there are no data in the literature concerning the polar dissociation cross-section for a water molecule.

\section{The Cost of Electron Impact Ionization of Water Molecule}

The determination of the ionization cost for atoms and molecules, both experimentally and theoretically, has a long history [46]. A lot of various models were proposed for the calculation of the ionization cost
$W$ in the case of monoenergetic beam $[6,47]$. They are based on the solution of the Fowler equation, the Spencer-Fano method of spectrum degradation, the Green-Barth method of consecutive generations, the Monte Carlo method, the continuous slowing down approximation (CSDA), and some others. The results of calculations for $W$ in the framework of several models were analyzed in work [47]. The analysis showed that the examined models give $W$-values which are close to that obtained for a hydrogen molecule.

In order to calculate the cost of water molecule ionization by a monoenergetic beam, a model developed on the basis of the CSDA, which was proposed in works $[48,49]$, will be used. In this model, the cost of electron impact ionization of the molecule with regard for the ionization by secondary electrons is determined by the formula

$W=\frac{E}{\sum_{n}\left\langle N_{i, n}\right\rangle}$,

where $E$ is the energy of primary electrons (reckoned in electronvolts), and $N_{i, n}(E)$ the number of ions generated by the $n$-th generation of electrons (the value $n=0$ stands for primary electrons). The number of ions generated by primary electrons equals

$N_{i, 0}=\int_{E_{\mathrm{iz}}}^{E} \frac{\sigma_{i}^{\mathrm{tot}}(E)}{L(E)} d E$,

where $\sigma_{i}^{\text {tot }}$ is the integral ionization cross-section $\left(\mathrm{cm}^{2}\right)$,

$L(E)=-\frac{1}{n} \frac{d E}{d x}$

is a function of the total energy loss $\left(\mathrm{cm}^{2} \mathrm{eV}\right), n$ the number of particles, and $d E / d x$ the stopping power. The function $L(E)$ is calculated by the formula

$$
\begin{aligned}
& L(E)=\frac{1}{n_{M} v} \frac{d E}{d t}+\sum \sigma_{\mathrm{ex}}(E) E_{\mathrm{ex}}+\sum \sigma_{\mathrm{iz}}(E) E_{\mathrm{iz}}+ \\
& +\sigma_{\mathrm{el}}(E) \frac{3 m_{e}}{m_{M}} E+\int_{0}^{\left(E-E_{\mathrm{iz}}\right) / 2} \varepsilon \sigma_{\mathrm{iz}}(E, \varepsilon) d \varepsilon
\end{aligned}
$$

where $\sigma_{\mathrm{el}}, \sigma_{\mathrm{ex}}$, and $\sigma_{\mathrm{iz}}$ are the cross-sections of elastic collisions, excitation, and ionization, respectively $\left(\mathrm{cm}^{2}\right) ; \sigma_{\mathrm{iz}}(E, \varepsilon)$ is the ionization differential crosssection $\left(\mathrm{cm}^{2} / \mathrm{eV}\right) ; \varepsilon$ the energy of secondary electrons; 
$n_{M}$ the concentration of neutrals $\left(\mathrm{cm}^{-3}\right) ; v$ the velocity of fast electrons $(\mathrm{cm} / \mathrm{s})$;

$\frac{d E}{d t}=\frac{2 \times 10^{-4} n_{e}^{0.97}}{E^{0.44}}\left(\frac{E-T_{e}}{E-0.53 T_{e}}\right)^{2.36}$

is the fast electron Coulomb energy loss [5]; $n_{e}$ the concentration of plasma electrons $\left(\mathrm{cm}^{-3}\right)$; and $T_{e}$ the average temperature of electrons in the plasma. The number of ions generated at the ionization by secondary electrons is determined as follows:

$$
\begin{aligned}
& N_{i, 1}=\int_{E_{\mathrm{iz}}}^{\left(E-E_{\mathrm{iz}}\right) / 2} n(E, \varepsilon) N_{i, 0}(\varepsilon) d \varepsilon, \\
& N_{i, 2}=\int_{3 E_{\mathrm{iz}}}^{\left(E-E_{i z}\right) / 2} n(E, \varepsilon) N_{i, 0}(\varepsilon) d \varepsilon .
\end{aligned}
$$

Here, $n(E, \varepsilon)$ is the number of secondary electrons with the energy $\varepsilon$ generated by primary electrons with the energy $E$, which is determined by the formula

$n(E, \varepsilon)=\int_{\varepsilon+E_{\mathrm{iz}}}^{E} \frac{\sigma(E, \varepsilon)}{L(E)} d E$.

In order to calculate the ionization cost for a water molecule, the data concerning the cross-sections of elementary processes, which were discussed in the previous section, were used. The differential cross-section of water ionization was determined in the framework of the binary-encounter-Bethe (BEB) model $[50,51]$. The corresponding calculated values are in a satisfactory agreement with the experimental data of work [52]. The average temperature of electrons in the plasma was selected to be lower than $1 \mathrm{eV}$, which corresponds to experimental data reported in work [8].

The results of calculation of the electron impact ionization cost for a water molecule neglecting the energy expenditure for Coulomb collisions are exhibited in Fig. 1. Figure 1 also illustrates the theoretical and experimental data for the cost of water molecule ionization taken from works [53-57]. One can see that the cost of water molecule ionization increases with a reduction of the electron energy. This behavior is quite predictable, because, as the electron energy decreases, the probability of the direct molecule ionization by the electron impact diminishes. The profile

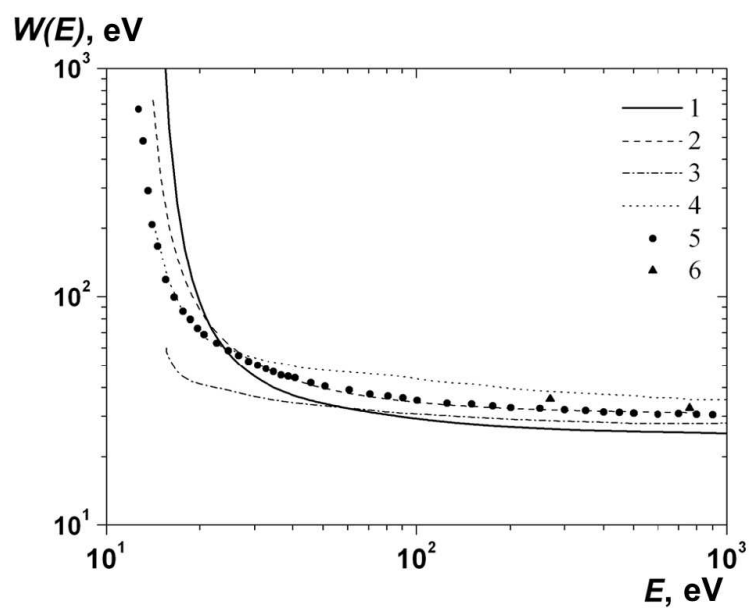

Fig. 1. Dependences of the electron impact ionization cost for a water molecule on the primary electron energy. Theoretical results: this work (1), the CSDA model (2) [53], the Monte Carlo model (3) [54] and (4) [55]. Experimental data: (5) [56] and $(6)[57]$

of the ionization cost curve is governed by the ionization cross-section. Therefore, if the contribution of only primary electrons were taken into consideration, the dependence of the ionization cost on the electron energy would have a minimum at the point, where the ionization cross-section is maximum. However, since the contribution of secondary electrons was also made allowance for, the ionization cost, after reaching a certain value, starts to change weakly as the electron energy grows further (see Fig. 1). Attention was attracted to this fact in the earlier works as well (see, e.g., works $[6,55])$.

As a result, there are two thresholds for the ionization cost values. In the first case, when the energy of primary electrons $E \rightarrow E_{\mathrm{iz}}$, the ionization cost $W \rightarrow \infty$. This circumstance is associated with the fact that the direct ionization of a neutral particle by the electron impact has a threshold character, and the ionization cross-section equals 0 at the electron energy $E=E_{\mathrm{iz}}[18,19]$. Accordingly, there is no contribution to the ion generation through the direct ionization by secondary and tertiary electrons at the energy of primary electrons $E \leq 2 E_{\mathrm{iz}}$ and $E \leq 3 E_{\mathrm{iz}}$, respectively. However, the ionization is possible due to other mechanisms, which were not taken into account in this calculation; e.g., cascade ionization. Therefore, the ionization cost at the electron energy $E=E_{\mathrm{iz}}$ has an appreciable and finite magnitude. In the second case where the energy of 


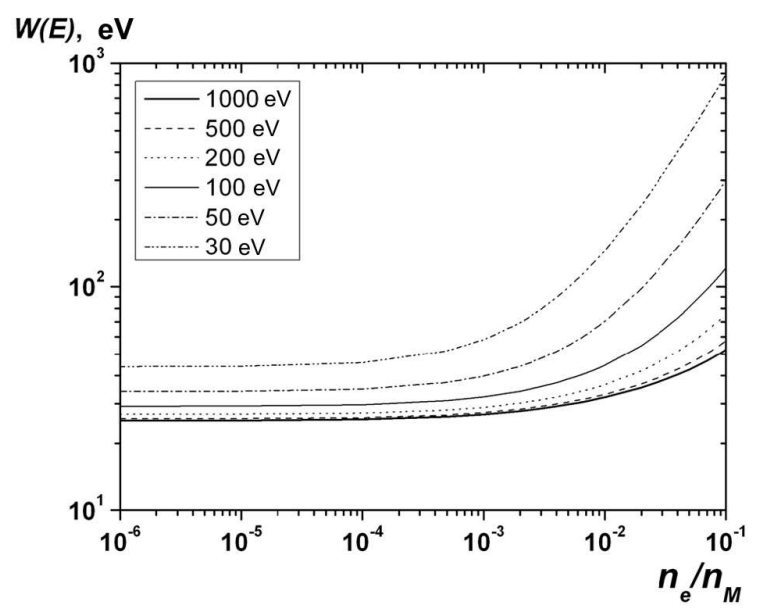

Fig. 2. Dependences of the electron impact ionization cost for a water molecule on the plasma ionization degree for various energies of primary electrons

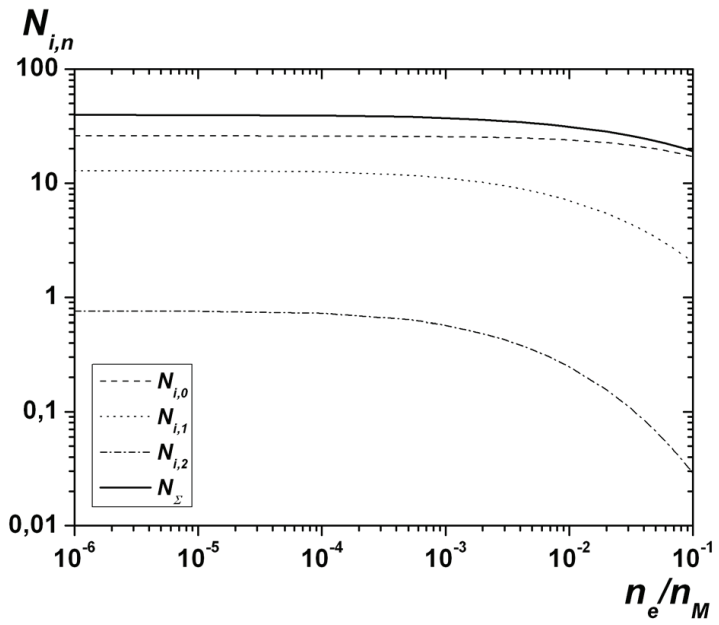

Fig. 3. Dependences of the average numbers of ions generated by primary, $N_{i, 0}$, secondary, $N_{i, 1}$, and tertiary, $N_{i, 2}$, electrons on the plasma ionization degree. The initial energy of primary electrons equals $1000 \mathrm{eV}$

primary electrons $E \gg 100 \mathrm{eV}$, the ionization cost $W \rightarrow$ const if the contribution of secondary, tertiary, and so on electrons in ionization cascades is taken into account.

In Fig. 2, the dependences of the cost of water molecule ionization by the electron impact on the plasma ionization degree are depicted. Here, in contrast to the previous works (see, e.g., works [53$55]$ ), Coulomb collisions were taken into consideration. Their contribution to the total value of ionization cost becomes appreciable at an ionization degree

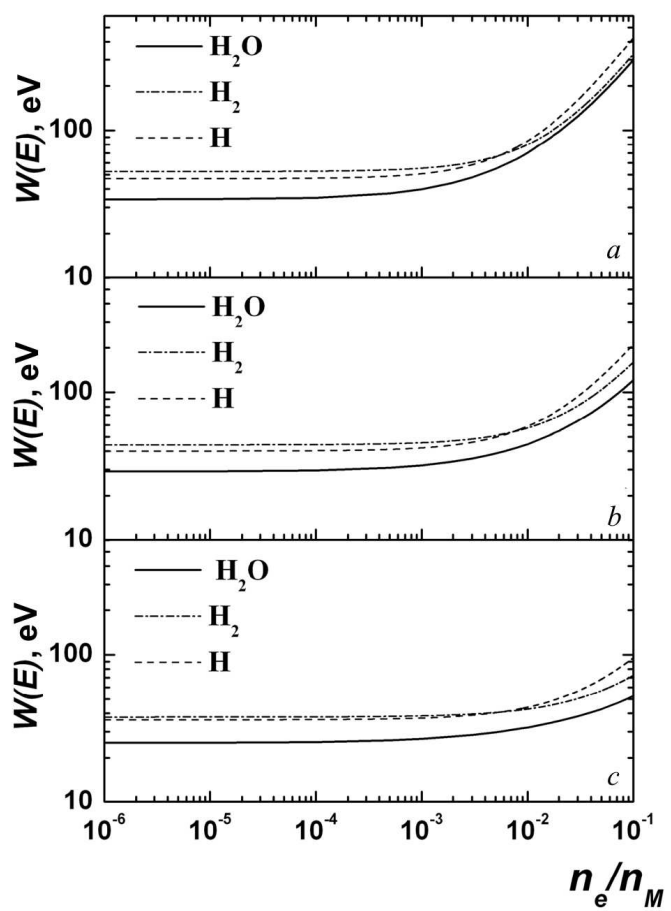

Fig. 4. Comparison of the dependences of the ionization cost for $\mathrm{H}_{2} \mathrm{O}$ (this work), $\mathrm{H}_{2}$, and $\mathrm{H}$ [20] on the ionization degree of a plasma-forming medium: $\mathrm{H}_{2} \mathrm{O}, \mathrm{H}_{2}$, and $\mathrm{H}$, respectively. The initial energy of primary electrons equals $50(a), 100(b)$, and $1000 \mathrm{eV}(c)$

of $10^{-4}$. As the ionization degree grows, this contribution to the ionization cost becomes more substantial: at relatively low energies of primary electrons $(\lesssim 200 \mathrm{eV})$, if the ionization degree is about $10^{-3}$, and at energies higher than $200 \mathrm{eV}$, if the ionization degree is about $10^{-2}$. For instance, at an energy of primary electrons of $1000 \mathrm{eV}$, the ionization cost value falls within the interval from 25.26 to $52.45 \mathrm{eV}$, if the ionization degree equals $0-0.1$.

In addition, the ionization degree growth is accompanied by a variation in the average number of ions and the relative contribution of ions generated at the direct ionization by primary, secondary, and tertiary electrons to the total ion number. As is seen from Fig. 3, at an ionization degree of $10^{-6}$ and a primary electron energy of $1000 \mathrm{eV}$, the contribution of primary electrons to the total number of generated ions amounts to $65.55 \%$, by secondary ones to $32.54 \%$, and by tertiary ones to $1.91 \%$. At an ionization degree of 0.1 , the corresponding contributions equal $89.25 \%$, $10.6 \%$, and $0.15 \%$, respectively; i.e. at higher ioniza- 
tion degrees, the contributions of secondary and tertiary electrons to ionization decrease.

\section{Discussion of the Results Obtained}

The weakly ionized molecular plasma contains molecules and molecular ions from the plasma-forming medium, as well as the products of the molecular dissociation (dissociative ionization) and plasmochemical reactions. This fact can substantially affect the final value of ionization cost, because the ionization cost values can differ considerably for different atoms (molecules, radicals). To confirm this statement, the ionization costs for $\mathrm{H}_{2} \mathrm{O}, \mathrm{H}_{2}$, and $\mathrm{H}$ are compared in Fig. 4. One can see that the ionization cost for $\mathrm{H}_{2} \mathrm{O}$ is about 1.3-1.5 times lower than those for $\mathrm{H}_{2}$ and H. In work [46], the influence of small impurities on the ionization cost in gases and the ionization cost for gas mixtures were considered. In particular, the ionization cost amounts to $30 \mathrm{eV}$ for He with an Ar admixture of $0.13 \%$, and to $42.7 \mathrm{eV}$ for pure He. In other words, the ionization cost depends on the plasma-forming medium. It should also be noted that water always contains various impurities, e.g., dissolved gas, so that the energy expenditure for their ionization will affect the final magnitude of ionization cost. Therefore, it is evident that the multicomponent composition of water has to be taken into account in further calculations, because it affects the value of energy expenditure for the plasma creation.

In works $[12,13]$, the ionization cost for a water molecule in the pulsed electric discharge was estimated on the basis of experimental data. The estimation showed that its magnitude falls within the interval from 222 to $92.6 \mathrm{eV}$ and depends on the energy of electrons in a plasma $(50-70 \mathrm{eV})$. Let us evaluate the degree of plasma ionization in those experiments. The experimentally measured electron concentration in a plasma amounts to $n_{e}=1.8 \times$ $\times 10^{13} \mathrm{~cm}^{-3}[12,13]$. Accepting the density of liquid water equal to $9.9821 \times 10^{-4} \mathrm{~kg} / \mathrm{cm}^{3}$ (at $T=$ $=20{ }^{\circ} \mathrm{C}$ and $p=0.1 \mathrm{MPa}$ ) [58], which corresponds to the concentration of neutral molecules $n_{M}=$ $=3.31 \times 10^{22} \mathrm{~cm}^{-3}$, the ionization degree amounts to $n_{e} / n_{M}=5.4 \times 10^{-10}$. For a saturated vapor at $T=100^{\circ} \mathrm{C}$ and $p=0.1 \mathrm{MPa}$, the density is equal to $5.9822 \times 10^{-7} \mathrm{~kg} / \mathrm{cm}^{3}$ [58], so that $n_{M}=$ $=1.986 \times 10^{19} \mathrm{~cm}^{-3}$ and $n_{e} / n_{M}=9.1 \times 10^{-7}$. Hence, the ionization degree of a plasma, by the order of
Table 2

\begin{tabular}{|r|l|r|c|}
\hline$E, \mathrm{eV}$ & $W_{0}, \mathrm{eV}$ & \multicolumn{1}{|c|}{$C$} & $\alpha$ \\
\hline 30 & 43.3 & 164.135 & 0.931 \\
50 & 34.04 & 57.968 & 0.875 \\
100 & 29.24 & 18.715 & 0.782 \\
200 & 26.95 & 9.052 & 0.708 \\
500 & 25.75 & 5.307 & 0.641 \\
1000 & 25.26 & 4.288 & 0.606 \\
\hline
\end{tabular}

magnitude, can amount to $10^{-6}$. A comparison of ionization cost for a water molecule estimated in works $[12,13]$ with the results of our calculations at an ionization degree of $10^{-6}$ shows that they diverge by a factor of 3 to 6.5. At an ionization degree of about $10^{-2}$, the difference between the estimated and calculated data amounts to $\pm 50 \%$, but this ionization degree of plasma can be unattainable experimentally $[12,13]$.

The discrepancy between the experimental and theoretical data is associated with several factors affecting their final values. Some those factors and their influence on the ionization cost were discussed above. Hence, the account of additional factors in further calculations and the performance of additional experiments should evidently allow a better agreement between the theoretical and experimental results to be obtained in the future.

\section{Conclusions}

The calculations carried out for the cost of water molecule ionization by a monoenergetic electron beam in the interval of primary electron energies of 15-1000 eV showed that the dependence of the water molecule ionization cost on the electron energy has a tendency to grow, as the electron energy diminishes. A model was considered, which considers the expenditure of the monoenergetic electron flux (beam) energy owing to Coulomb collisions at the beam passage through a weakly ionized plasma. The corresponding calculations show that the ionization cost for a water molecule grows with the plasma ionization degree. The calculated dependence of the water molecule ionization cost on the water ionization degree within an interval from 0 to 0.1 and at an energy of primary electrons of $1000 \mathrm{eV}$ testifies that this parameter grows from 25.26 to $52.45 \mathrm{eV}$ in the examined interval. 


\section{APPENDIX}

The theoretical values of ionization cost for the water molecule obtained in this work were approximated by the following empirical expression proposed in work [20]:

$W(x)=W_{0}\left(1+C x^{\alpha}\right)$,

where $x=n_{e} / n_{M}$ is the ionization degree, $W_{0}$ the ionization cost at $x=0$, and $C$ and $\alpha$ are fitting parameters. The values of $W_{0}, C$, and $\alpha$ for various $E$-values are shown in Table 2 .

1. M. Larsson, W.D. Geppert, and G. Nyman, Rep. Prog. Phys. 75, 066901 (2012).

2. D.W. Savin, N.S. Brickhouse, J.J. Cowan et al., Rep. Prog. Phys. 75, 036901 (2012).

3. R.K. Hobbie and B.J. Roth, Intermediate Physics for Medicine and Biology (Springer, New York, 2007).

4. V.M. Byakov and S.V. Stepanov, Usp. Fiz. Nauk 176, 487 (2006).

5. D.X. Liu, P. Bruggeman, F. Iza et al., Plasma Sources Sci. Technol. 19, 025018 (2010).

6. G.D. Alkhazov, Zh. Tekhn. Fiz. 41, 2513 (1971).

7. S. Samukawa, M. Hori, S. Rauf et al., J. Phys. D. 45, 253001 (2012)

8. P. Bruggeman and C. Leys, J. Phys. D. 42, 053001 (2009).

9. Y. Yang, A. Fridman, and Y.I. Cho, Adv. Heat Transf. 42, 179 (2010).

10. A.A. General and Yu.O. Shpenyk, Ukr. Fiz. Zh. 58, 116 (2013).

11. E.I. Skibenko, V.B. Yuferov, I.V. Buravilov et al., Ukr. J. Phys. 53, 356 (2008).

12. E.I. Skibenko, Yu.V. Kovtun, A.I. Skibenko, and V.B. Yuferov, in Proceedings of the 15-th International Conference on Physics of Pulse Discharges in Condensed Media, Mykolaiv (2011), p. 70 (in Russian).

13. E.I. Skibenko, Yu.V. Kovtun, A.I. Skibenko, and V.B. Yuferov, Tech. Phys. 57, 188 (2012).

14. Yu.V. Kovtun, E.I. Skibenko, and V.B. Yuferov, in Proceedings of the 16-th International Conference on Physics of Pulse Discharges in Condensed Media, Mykolaiv (2013), p. 30 (in Russian).

15. V.Ya. Ushakov, V.F. Klimkin, and S.M. Korobeynikov, Impulse Breakdown of Liquids (Springer, Berlin, 2007).

16. Y. Itikawa and N. Mason, J. Phys. Chem. Ref. Data 34, 1 (2005)

17. C.G. Elles, A.E. Jailaubekov, R.A. Crowell, and S.E. Bradforth, J. Chem. Phys. 125, 044515 (2006).

18. J.B. Hasted, Physics of Atomic Collisions (Butterworths, London, 1964).

19. H.S.W. Massey and E.H.S. Burhop, Electronic and Ionic Impact Phenomena (Clarendon Press, Oxford, 1952).

20. A. Dalgarno, Min Yan, and Weihong Liu, Astrophys. J. Suppl. 125, 237 (1999).
21. A. Dalgarno and G. Lejeune, Planet. Space Sci. 19, 1653 (1971)

22. J.L. Fox, A. Dalgarno, and G.A. Victor, Planet. Space Sci. 25, 71 (1977).

23. T.E. Cravens, G.A. Victor, and A. Dalgarno, Planet. Space Sci. 23, 1059 (1975).

24. J.L. Fox and A. Dalgarno, Planet. Space Sci. 27, 491 (1979).

25. J.L. Fox and G.A. Victor, Planet. Space Sci. 36, 329 (1988).

26. Weihong Liu and G.A. Victor, Astrophys. J. 435, 909 (1994).

27. T. Shirai, T. Tabata, and H. Tawara, At. Data Nucl. Data Tabl. 79, 143 (2001)

28. Y. Itikawa and N. Mason, Phys. Rep. 414, 1 (2005).

29. J. Tennyson, N.F. Zobov, R. Williamson, and O.L. Polyansky, J. Phys. Chem. Ref. Data. 30, 735 (2001).

30. M.A. Khakoo, C. Winstead, and V. McKoy, Phys. Rev. A 79, 052711 (2009).

31. P.A. Thorn, M.J. Brunger, P.J.O. Teubner et al., J. Chem. Phys. 126, 064306 (2007).

32. P.A. Thorn, M.J. Brunger, H. Kato et al., J. Phys. B 40, 697 (2007).

33. P. Thorn, L. Campbell, and M. Brunger, PMC Physics B 2, 1 (2009).

34. W. Lotz, Z. Phys. 206, 205 (1967).

35. S.Y. Truong, A.J. Yencha, A.M. Juarez et al., Chem. Phys. 355, 183 (2009).

36. S.Y. Truong, A.J. Yencha, A.M. Juares et al., Chem. Phys. Lett. 474, 41 (2009).

37. T. Harb, W. Kedzierski, and J.W. McConkey, J. Chem. Phys. 115, 5507 (2001).

38. Kaijun Yuan, Lina Cheng, Yuan Cheng et al., J. Chem. Phys. 131, 074301 (2009).

39. A.N. Zavilopulo, F.F. Chipaev, and O.B. Shpenik, Zh. Tekhn. Fiz. 75, 19 (2005).

40. S.W.J. Scully, J.A. Wyer,V. Senthil et al., Phys. Rev. A 73, 040701 (2006).

41. H. Sann, T. Jahnke, T. Havermeier et al., Phys. Rev. Lett.106, 133001 (2011).

42. H.B. Pedersen, C. Domesle, L. Lammich et al., Phys. Rev. A 87, 013402 (2013).

43. F. Fremont, C. Leclercq, A. Hajaji et al., Phys. Rev. A 72 042702 (2005).

44. S.J. King and S.D. Price, Int. J. Mass Spectrom. 277, 84 (2008).

45. D.J. Haxto, C.W. McCurdy, and T.N. Rescigno, Phys. Rev. A 75, 012710 (2007).

46. J.M. Valentine and S.C. Curran, Rep. Prog. Phys. 21, 1 (1958).

47. R.H. Garvey and A.E.S. Green, Phys. Rev. A 14, 946 (1976).

48. S.P. Khare, J. Phys. B 3, 971 (1970).

49. S.P. Khare, Rad. Res. 64, 106 (1975).

50. Y.-K. Kim and M.E. Rudd, Phys. Rev. A 50, 3954 (1994)

ISSN 2071-0186. Ukr. J. Phys. 2016. Vol. 61, No. 1 
51. W. Hwanga, Y.-K. Kim, and M.E. Rudd, J. Chem. Phys. 104, 2956 (1996).

52. M.A. Bolorizadeh and M.E. Rudd, Phys. Rev. A 33, 882 (1986).

53. G.J. Kutcher and A.E.S. Green, Rad. Res. 67, 408 (1976).

54. C. Champion, Phys. Med. Biol. 48, 2147 (2003).

55. J.A. La Verne and A. Mozumder, Rad. Res. 131, 1 (1992).

56. D. Combecher, Rad. Res. 84, 189 (1980).

57. G.P. Stonell, M. Marshall, and J.A. Simmon, Rad. Res. 136, 341 (1993).

58. D.R. Lide, CRC Handbook of Chemistry and Physics (CRC Press, Taylor and Francis, Boca Raton, FL, 2010).

Received 22.01.15.

Translated from Ukrainian by O.I. Voitenko
Ю.В. Ковтун

ЕНЕРГЕТИЧНІ ВИТРАТИ НА ІОНІЗАЦІЮ

МОЛЕКУЛИ ВОДИ ЕЛЕКТРОННИМ УДАРОМ

У СЛАБКОІОНІЗОВАНІЙ ПЛАЗМІ

$\mathrm{P}$ е $з$ ю м е

Проведено розрахунки ціни іонізації молекули води моноенергетичним пучком у діапазоні енергії первинних електронів 15-1000 еВ. Отримано залежності ціни іонізації від ступеня іонізації води від 0 до 0,1. Показано, що ціна іонізації зростає із збільшенням ступеня іонізації та для енергії первинних електронів 1000 еВ вона зростає від 25,26 еВ до 52,45 еВ у разрахунковому інтервалі ступеня іонізації. 